\title{
Internal plasma potential profiles in a laboratory-model Hall thruster
}

\author{
James M. Haas and Alec D. Gallimore \\ Plasmadynamics and Electric Propulsion Laboratory, Department of Aerospace Engineering, \\ The University of Michigan, College of Engineering, Ann Arbor, Michigan 48109
}

(Received 18 April 2000; accepted 9 November 2000)

\begin{abstract}
The Plasmadynamics and Electric Propulsion Laboratory High-speed Axial Reciprocating Probe system is used in conjunction with a floating emissive probe to measure plasma potential in the discharge chamber of the P5 Hall thruster. Plasma potential measurements are made at a constant voltage, $300 \mathrm{~V}$, at two different discharge current conditions: 5.4 and $10 \mathrm{~A}$. The plasma potential contours for the 5.4 A case indicate that the acceleration region begins several millimeters upstream of the exit plane, extends several centimeters downstream, and is uniform across the width of the discharge chamber. The $10 \mathrm{~A}$ case is similar to the $5.4 \mathrm{~A}$ case with the exception that the acceleration region is shifted downstream on centerline. Axial electric field profiles, computed from the measured potential, show a double peak structure in the $5.4 \mathrm{~A}$ case, indicating a zone of ion deceleration. Perturbations to the discharge current are shown to correspond spatially with the location of the peak electric field indicating that thruster perturbations may result from a disturbance to the Hall current, as opposed to ablation of probe material. This conclusion is supported by the lack of any observable material ablation. (C) 2001 American Institute of Physics.
\end{abstract}

[DOI: $10.1063 / 1.1338535]$

\section{INTRODUCTION}

The Hall thruster, also referred to as a closed drift thruster (CDT), is a coaxial device in which orthogonal electric and magnetic fields are employed to ionize an inert gas, such as xenon, and accelerate the resulting ions to produce thrust. The main components of the CDT are shown in Fig. 1. The magnetic field that is produced by an electromagnet is channeled between an inner ferromagnetic core (pole piece) and outer ferromagnetic ring. The electromagnet circuit is typically composed of a single central magnet coil in parallel with a series of outer magnet coils, the number of which may range from one to eight, depending on the particular thruster. In the case of low power thrusters, permanent magnets have also been used. Though separate power supplies can be used to energize the electromagnet coils, the number of turns in each is usually set so the electromagnet establishes the proper magnetic field strength when it is operated in series with the discharge supply. This configuration results in an approximately radial magnetic field with a peak strength of a few hundred Gauss near the exit of the engine. The field strength is such that only the electrons are magnetized. The radial magnetic field exhibits a bell-shaped axial profile with the peak situated near the exit. Most of the acceleration takes place near the region of peak magnetic field strength.

An axial electric field is provided by applying a voltage between the anode and the cathode. As the electrons stream upstream toward the anode from the cathode, their motion is impeded by the magnetic field. Because of the orientation of the electric and magnetic fields, the electrons drift in the $\mathbf{E} \times \mathbf{B}$ direction, forming an azimuthal Hall current. These electrons also ionize propellant molecules that are injected through the anode and which are subsequently accelerated by the axial electric field. This emerging ion flux is then neu- tralized by electrons from the external cathode. From an electrostatic point of view, the magnetic field suppresses the axial mobility of the electrons while exerting essentially no effect on the ions. This enables the plasma to support an axial electric field with a potential difference close to the applied voltage between the electrodes. Thus, the bulk of the ions are accelerated to kinetic energies within $80 \%$ of the applied discharge voltage. Moreover, the mixture of electrons and ions in the acceleration zone means that the plasma is electrically neutral, and as such, is not space-charge limited in ion current density as in the case of a gridded ion thruster. This combination of processes accounts for the CDT's high thrust efficiency and thrust density. The electromagnetic point of view is simply that the azimuthal Hall current interacts with the radial applied magnetic field and accelerates the plasma axially through the $\mathbf{J} \times \mathbf{B}$ Lorentz force.

The role of Hall thrusters in spacecraft propulsion is constantly evolving and expanding as new missions, covering the full spectrum of spacecraft size and power, continue to be developed. A large number of such missions can significantly benefit from the unique performance characteristics of Hall thrusters. These range from sub-kW thrusters for small satellites, to $1.5 \mathrm{~kW}$ thrusters for station-keeping, to high power thrusters for orbit raising/transfer applications. ${ }^{1-4}$

While a number of Hall thrusters have been, or are in the process of being, flight qualified ${ }^{5,6}$ many of the basic physical processes inside the Hall thruster are still not fully understood. In order to improve the performance of the next generation of thrusters, as well as to address spacecraft interaction issues, the ionization and acceleration mechanisms inside the discharge chamber of the Hall thruster must be better understood. Toward this end, the Plasmadynamics and Electric Propulsion Laboratory (PEPL) at the University 


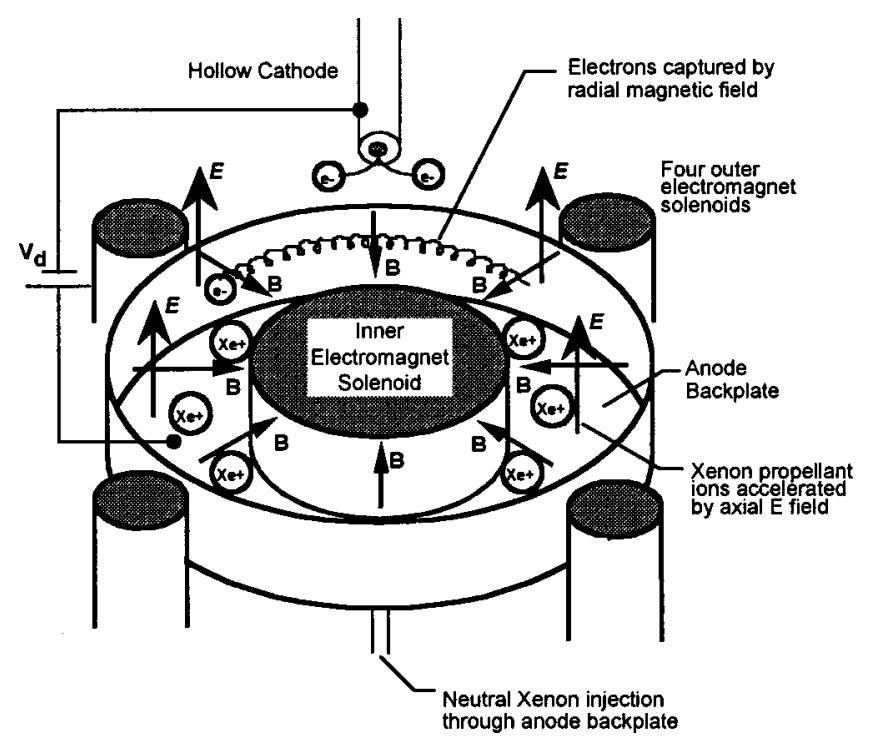

FIG. 1. Schematic showing typical CDT construction.

of Michigan has constructed a laboratory model Hall thruster, designated the P5, designed specifically to facilitate measurements in the discharge chamber. ${ }^{7}$ As part of the same program, PEPL has constructed and successfully demonstrated a High-speed Axial Reciprocating Probe $^{8}$ (HARP) system to allow electrostatic probe measurements without significantly perturbing thruster operation. This is accomplished by inserting and removing probes on time scales such that the probe does not heat to the point where probe material is ablated.

In addition to providing direct insight on the ionization and acceleration mechanisms, data on the internal plasma structure will play a valuable role in validating existing and future numerical models. ${ }^{9,10}$ The increasing time and expense associated with developing new thrusters makes it imperative that accurate and reliable predictive models are developed.

\section{OBJECTIVE}

The objective of this research was to measure the plasma potential inside the discharge chamber of a Hall thruster while avoiding significant perturbation to thruster operation. The resulting data were used to determine the magnitude and spatial structure of the acceleration region as well as to better understand the role of the Hall current in thruster operation.

\section{EXPERIMENTAL SETUP}

\section{A. Vacuum chamber}

All experiments were conducted in the University of Michigan's 6-m-diam by 9-m-long Large Vacuum Test Facility. The pumping system consists of four CVI model TM1200 Re-Entrant Cryopumps providing a measured xenon pumping speed of $140000 \mathrm{l} / \mathrm{s}$. The ultimate base pressure of the facility is less than $2 \times 10^{-7}$ Torr. Thruster discharge voltage was fixed at $300 \mathrm{~V}$ while two discharge current settings were investigated: 5.4 and $10 \mathrm{~A}$. These current settings corresponded to anode xenon mass flow rates of 63 and 112

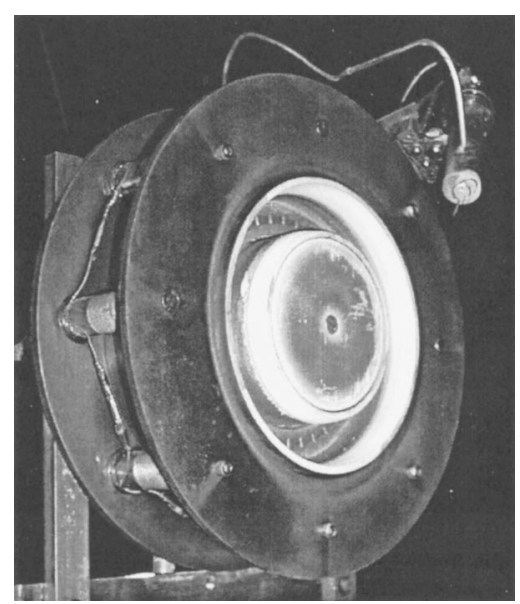

FIG. 2. The UM/AFRL P5 laboratory-model Hall thruster.

sccm, respectively. The cathode mass flow rate remained constant at $6 \mathrm{sccm}$. Chamber pressure was measured using a Varian nude hot filament vacuum gauge calibrated for N2 and gave an indicated pressure of $5.5 \times 10^{-6}$ Torr $(5.4 \mathrm{~A})$ and $9.6 \times 10^{-6}$ Torr $(10 \mathrm{~A})$ when corrected for xenon. Details of the facility have been presented in a previous work. ${ }^{7}$

\section{B. Thruster}

The thruster used was the University of Michigan/Air Force Research Laboratory P5 $5 \mathrm{~kW}$ laboratory model Hall thruster, shown in Fig. 2. The diameter of the outer wall of the discharge chamber is $170 \mathrm{~mm}$ with a width and depth of $25.4 \mathrm{~mm}$ and $38 \mathrm{~mm}$, respectively. This thruster was developed specifically for diagnostic access to the discharge chamber. Compared to smaller thrusters, the P5 provides a larger discharge chamber for better spatial resolution for electrostatic probes as well as a lower power density to reduce heat flux to the probe. Details of the P5 construction, as well as characterization of its thrust, specific impulse, and efficiency have been reported, along with plasma parameter profiles in the plume, in a previous work. ${ }^{7}$ The P5 incorporates a lanthanum hexaboride $\left(\mathrm{LaB}_{6}\right)$ cathode.

\section{Emissive probe}

Inside the Hall thruster, the flux of high-energy particles sputters and/or heats electrostatic probe material causing it to ablate. Local plasma characteristics are then affected through emission of relatively cold probe material. These local variations propagate through the plasma, perturbing thruster operation, making it imperative that the residence time of any diagnostic inside the Hall thruster be minimized. It is for this reason that the floating emissive probe was chosen to make plasma potential measurements inside the P5. The floating emissive probe provides a direct measure of plasma potential without the requirement of a voltage sweep or data reduction operations, as is the case for both the standard emissive probe and the Langmuir (electron-collection) probe.

While the floating emissive probe offers many advantages, there are limitations that must be taken into account. Specifically, the presence of a magnetic field and large den- 


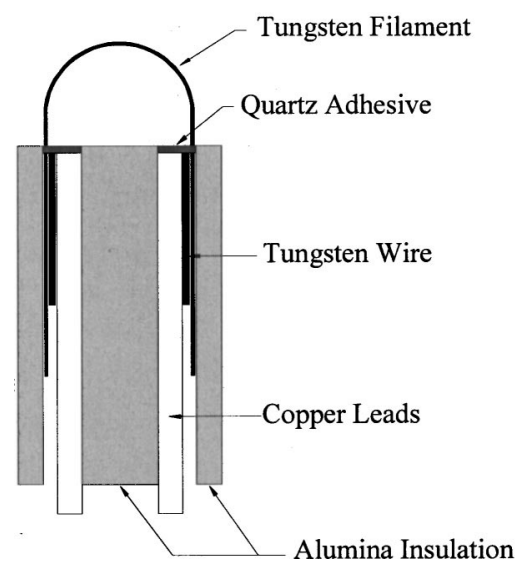

FIG. 3. Schematic of the emissive probe showing its individual parts and construction. When heated to the point where electrons are emitted, the probe floats at the local plasma potential.

sity gradients may result in space-charge effects, causing significant variation between the potential of the emitting probe and the actual plasma potential. In the case of the P5, the magnetic field is sufficiently weak that space-charge effects can be avoided by sizing the probe such that the probe wire diameter is much less than the electron gyroradius. Hershkowitz ${ }^{11}$ indicated this condition for a probe with diameter $d$ as $\mathrm{B}[\mathrm{G}] \ll 4.8^{*}(\mathrm{Tev})^{1 / 2} / \mathrm{d}$. The emissive probe used in the P5 had a diameter of $0.0127 \mathrm{~cm}$ while the electron temperature was estimated to be approximately $40 \mathrm{eV}$, corresponding to the peak in the magnetic field. This resulted in the condition $\mathrm{B}[\mathrm{G}] \ll 2300$. The magnetic field in the P5 peaked at $200 \mathrm{G}$, indicating that space-charge effects were negligible.

Figure 3 shows a schematic of the probe construction. The emitting portion of the probe was a filament made from 0.127 -mm-diam tungsten wire. The ends of this filament were inserted approximately $76 \mathrm{~mm}$ down a $152 \mathrm{~mm}$ length of double bore alumina tubing along with 30 AWG copper wire leads. The outer diameter of the alumina tubing was 1.5 $\mathrm{mm}$. Once the tungsten filament and copper leads were in place additional, shorter lengths of tungsten wire were inserted into the alumina tubing to provide a tight fit and guarantee good contact between the tungsten and copper wires. The width and height of the filament when mounted in the alumina insulator was approximately $1 \mathrm{~mm}$. The probe was oriented such that the alumina insulator was aligned with the axis of the thruster and the plane of the loop of the probe filament was normal to the thruster radial direction.

The theory of the floating emissive probe is well established and relatively straightforward to implement. ${ }^{12}$ The circuit consisted of the emissive probe, a floating power supply capable of supplying enough current to heat the filament (4-7 A), and a voltage meter. Figure 4 shows a schematic of the probe circuit. The sampling rate of the oscilloscope was dictated by the total sweep time of the probe and was set at 1 ms. This resulted in aliasing of the signal so that high frequency oscillations in the $10-30 \mathrm{kHz}$ range, typical of the Hall thruster, could not be resolved. Therefore, the data presented constitute a "time-averaged" plasma potential. The

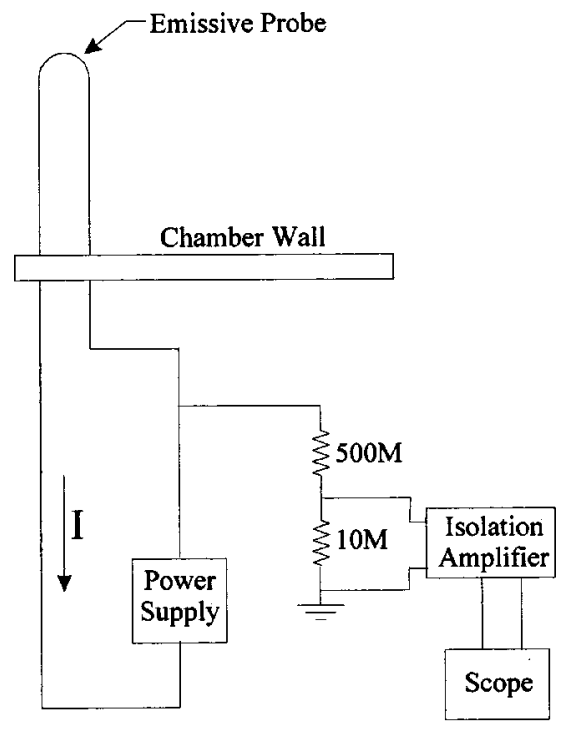

FIG. 4. The floating emissive probe circuit consists of a current source to heat the filament and an oscilloscope for measuring the resulting floating/ plasma potential.

correct heater current was determined by taking several preliminary sweeps and observing when the probe potential saturated. As stated previously, electrostatic probes are heated considerably by the flux of high-energy particles in the discharge channel of the Hall thruster. One consequence of this effect is that when the emissive probe is inserted into the thruster with insufficient power to reach the saturation emissive current, the plasma provides additional filament heating and the probe potential continues to increase when the probe is stationary. By increasing the heater current on each subsequent sweep and observing when the potential remained constant while probe position was fixed inside the thruster, a sufficient heater current was determined. Figure 5

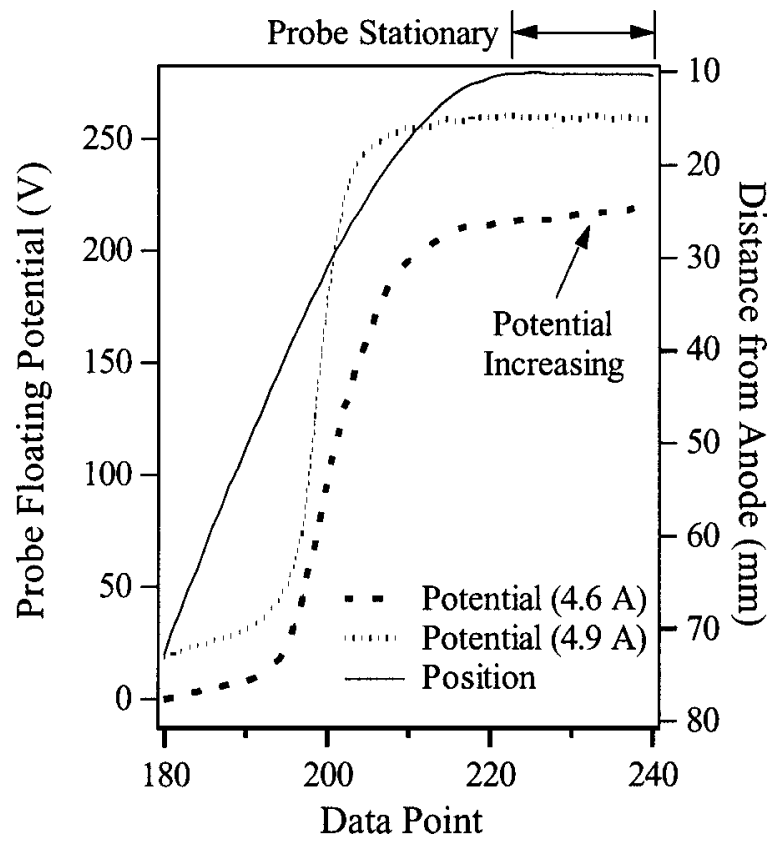

FIG. 5. Plot showing floating potential at heater currents of 4.6 and 4.9 A. At $4.6 \mathrm{~A}$, the probe potential continues to rise while stationary. 
shows the difference between sufficient and insufficient heater current.

A floating power supply provided current to heat the filament to the point where it was thermionically emitting sufficient electrons to neutralize the sheath. At this point, the probe was theoretically floating at the local plasma potential. The power supply was not perfectly isolated from ground, however, which introduced the possibility of significant leakage current when the circuit floated at high potential. This could result in the circuit floating at a potential less than the true potential. To determine the effect of this leakage current, the measured plasma potential near the anode was compared to the true anode potential. The excellent agreement between the two $(<2 \mathrm{~V}$ difference) indicated that the effect of leakage current was negligible. The heater current varied between tests due to slight variations in individual probe designs, ranging between 4.5 and $6.0 \mathrm{~A}$. The probe potential was divided and sent through an isolation amplifier capable of floating its input as high as $2500 \mathrm{~V}$. Because the heater current remained on during the duration of the measurement, a voltage drop existed across the tungsten filament. This value remained below $5 \mathrm{~V}$ for each data sweep, and contributed an estimated uncertainty of approximately $\pm 2.5 \mathrm{~V}$. The fragility of the emissive probe when swept at high speed made it very difficult to perform multiple measurements. However, two sets of data were collected for each axial sweep (during insertion and removal of the probe) providing an estimate of the reproducibility of the data. Analysis of both sets of data indicated an average standard deviation of less than $4 \%$. An analysis by Hargus ${ }^{13}$ of the change in apparent potential due to the presence of the heater current indicated an uncertainty of approximately $-3 /+6 \mathrm{~V}$. Coaxial cables were used for the entire circuit, both inside and outside the chamber, to reduce noise. In addition, isolated feedthroughs allowed every component of the circuit to be grounded at a single point to minimize noise pick-up through ground loops.

\section{Positioning system}

The emissive probe was positioned inside the Hall thruster discharge chamber using the PEPL HARP system. The HARP system allowed the probe to be inserted into, and removed from, the thruster on a time scale under $100 \mathrm{~ms}$. This allowed measurements to be made with very little perturbation to thruster operation, the extent of which was determined by monitoring the discharge current during probe movement. Previous attempts to interrogate the interior of a Hall thruster using electrostatic probes have caused the discharge current to vary by as much as $150 \% .{ }^{13}$ Use of the emissive probe with the HARP system caused a maximum perturbation of $15 \%$ of the nominal discharge current value but was less than $10 \%$ for the majority of measurements. Potential data were taken during both insertion and removal of the probe and averaged to get a final potential value. Figure 6 shows the cross-sectional area inside the discharge chamber where plasma potential was measured.

Radial movement was accomplished by mounting the thruster on a linear table. Between axial sweeps with the HARP system, the thruster was moved radially such that a

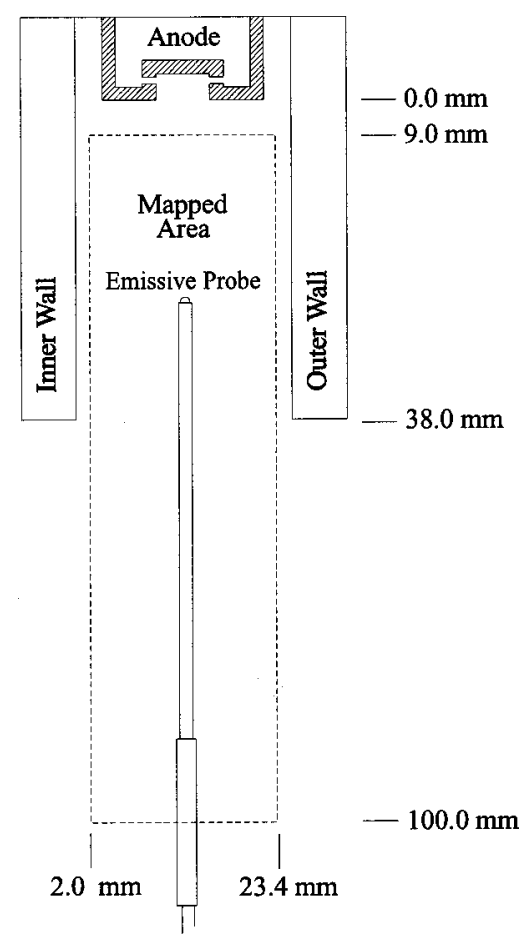

FIG. 6. The area mapped begins approximately $9 \mathrm{~mm}$ from the anode face, extends $10 \mathrm{~cm}$ downstream of the exit plane, and comes to within $2 \mathrm{~mm}$ of the inner and outer walls of the discharge chamber. Emissive probe is shown rotated $90^{\circ}$ about its axis.

two-dimensional cross section of the discharge chamber was covered. Note that the axial position throughout this paper corresponds to the tip of the filament.

\section{RESULTS AND DISCUSSION}

Plasma potential measurements with respect to ground were made at a constant voltage, $300 \mathrm{~V}$, at discharge current settings of 5.4 and $10 \mathrm{~A}$. For the two cases considered here, the magnetic field profile (both magnitude and shape) was kept constant and only the mass flow rate was adjusted. As discussed in Sec. I, the magnetic field plays an important role in that it impedes electron motion toward the anode, enabling the plasma to sustain an electric field. The maximum electric field should then occur near the magnetic field peak. In practice, the two peaks are not spatially concomitant due to other plasma effects, such as electron pressure. Figure 7 shows the axial and radial magnetic field profiles for reference.

Figure 8 shows three representative potential profiles at $300 \mathrm{~V}, 5.4 \mathrm{~A}$ : on centerline and along the inner and outer walls. For this case, the cathode potential with respect to ground was $-21 \mathrm{~V}$, therefore the anode potential was $279 \mathrm{~V}$ with respect to ground. Plasma potential profiles for the 5.4 A case show that the potential remains nearly constant over the first $75 \%$ of the channel. As expected, a sharp drop occurs between 30 and $40 \mathrm{~mm}$, indicative of the location of the main acceleration region. The axial electric field profiles show clearly the location of maximum acceleration at 35.5 $\mathrm{mm}, 2.5 \mathrm{~mm}$ upstream of the exit plane. The data indicate that the acceleration region extends $2-3 \mathrm{~cm}$ downstream of the exit plane, which agrees quite well with independent 


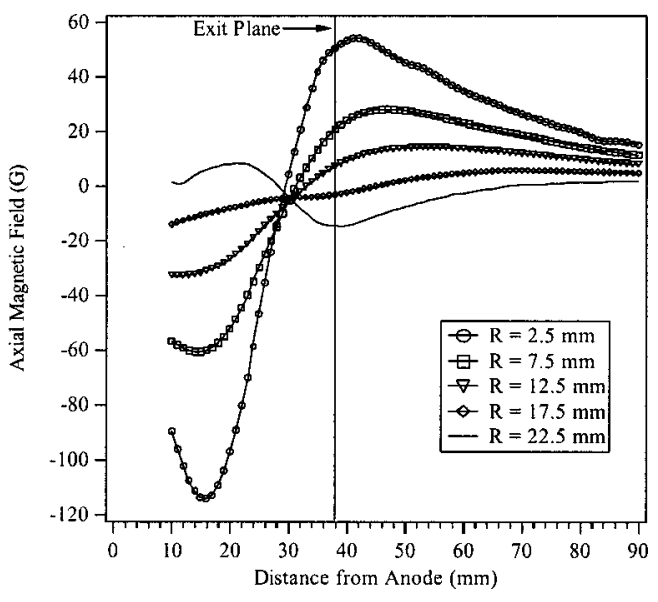

a) Axial magnetic field.

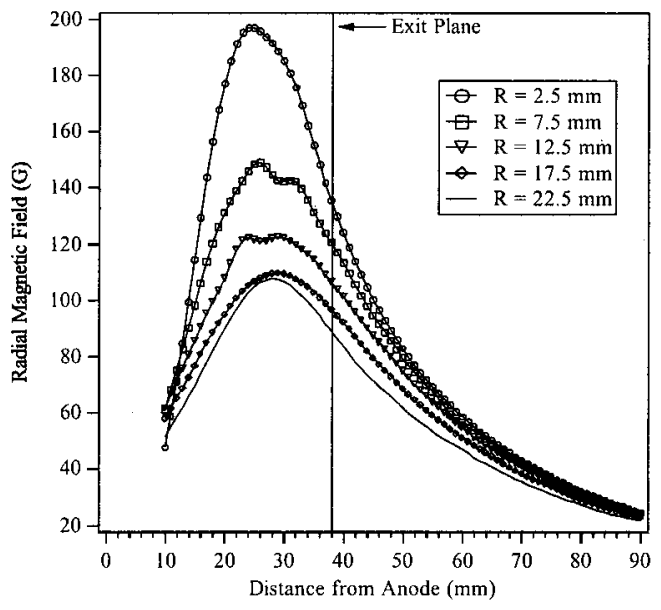

b) Radial magnetic field

FIG. 7. Axial profiles of the (a) axial and (b) radial magnetic field in the thruster discharge channel.

laser-induced fluorescence (LIF) data taken by Williams ${ }^{14}$ on the same thruster at the same conditions. These LIF measurements were made at axial positions 1 and $100 \mathrm{~mm}$ downstream of the exit plane and indicate ion speeds of 11000 and $16000 \mathrm{~m} / \mathrm{s}$, respectively. This speed increase corresponds to an accelerating potential of approximately $90 \mathrm{~V}$. Referring to Fig. 8, plasma potential at the exit plane is 100 $\mathrm{V}$ and $10 \mathrm{~cm}$ downstream (not shown in the figure) decreases to approximately $15 \mathrm{~V}$. Thus the accelerating potential as measured by LIF $(90 \mathrm{~V})$ agrees with that measured by the emissive probe $(85 \mathrm{~V})$ within $5 \%$. This large potential drop outside the thruster has also been observed with the Stanford low-power Hall thruster. ${ }^{15}$ Note that the potential profile remains uniform across the width of the discharge chamber, which can be seen clearly in Fig. 8. LIF data from the P5 show the same uniformity. One additional feature of the potential data that bears further scrutiny is the plateau that occurs approximately $5 \mathrm{~mm}$ downstream of the exit plane, most prominently seen on the inner wall profile. This will be discussed further in the following text.

Potential data were also taken at a higher power level to

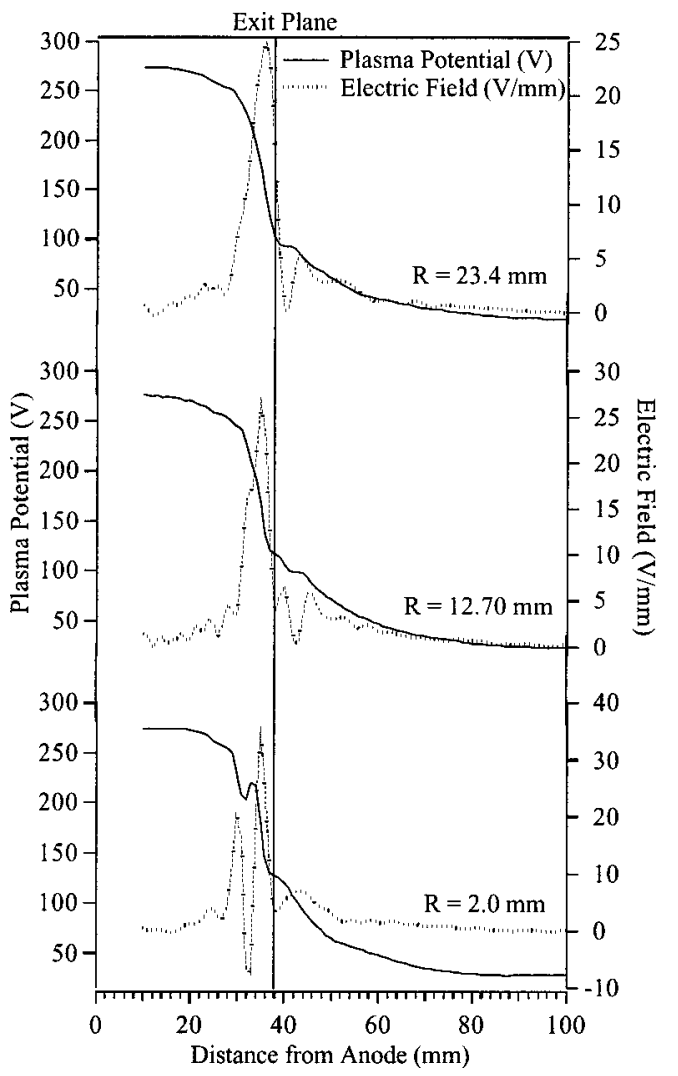

FIG. 8. Plasma potential and axial electric field profiles at three representative radial locations for the $300 \mathrm{~V}, 5.4 \mathrm{~A}$ case. The anode face corresponds to a position of $0 \mathrm{~mm}$ and the exit plane, as marked, is at a position of 38 $\mathrm{mm}$.

study the evolution of the potential structure. Figure 9 shows plasma potential profiles at $300 \mathrm{~V}, 10 \mathrm{~A}$ at the same three radial positions as in Fig. 8. Cathode and anode potentials with respect to ground were -23 and $277 \mathrm{~V}$, respectively. These data are very similar to the $5.4 \mathrm{~A}$ case. The potential remains approximately constant, at anode potential, over the first $75 \%$ of the discharge channel. The acceleration region begins near the exit plane and extends several centimeters downstream. LIF $^{14}$ data at these conditions again show a significant acceleration downstream of the exit plane $(\sim 100$ $\mathrm{V})$, however, they fail to reflect the lack of radial uniformity in the downstream accelerating potential which varies from approximately $75 \mathrm{~V}$ along the outer wall, to $150 \mathrm{~V}$ along the inner wall, to $180 \mathrm{~V}$ on the channel center-line. Referring again to Fig. 9, a significant feature of the $10 \mathrm{~A}$ data is that the axial electric field magnitude is nearly $25 \%$ percent lower, indicating a longer acceleration region. Baranov ${ }^{16}$ has shown that the length of the acceleration layer depends on both the radial magnetic field profile and electron temperature. The increase in mass flow rate effectively increases the ionization collision frequency, lowering the energy of the electrons since the ionization process is a loss mechanism. The result is a lower temperature gradient, therefore, lower electric field. The reduction of temperature gradients with increasing discharge current is predicted by the onedimensional code of Ahedo ${ }^{17}$ and is consistent with the data in Fig. 9. 


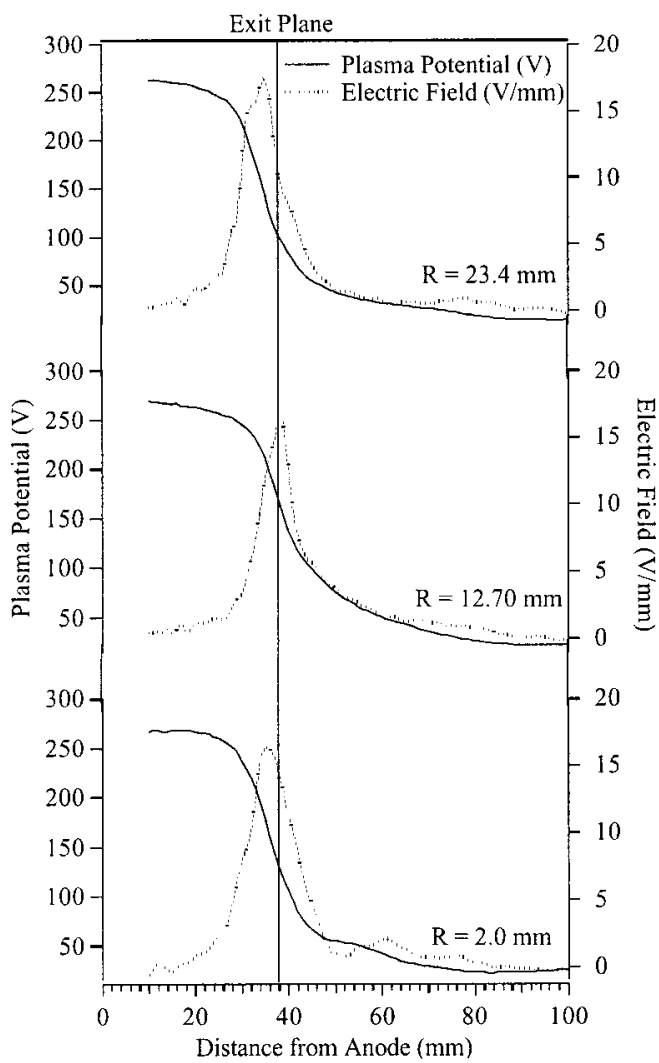

FIG. 9. Plasma potential and axial electric field profiles at three representative radial locations for the $300 \mathrm{~V}, 10 \mathrm{~A}$ case. The anode face corresponds to a position of $0 \mathrm{~mm}$ and the exit plane, as marked, is at a position of $38 \mathrm{~mm}$.

Returning to the radial nonuniformity of the potential profiles, note that the axial electric field peak along the outer wall occurs 2-3 mm upstream of the exit plane as in the 5.4 A case. Along the inner wall, the peak is shifted forward but still occurs upstream of the exit plane. The most significant difference between the two cases is seen in the centerline data where the peak electric field is shifted to a position downstream of the exit plane. As with the axial electric field magnitude, since the magnetic field is unchanged, this implies a shift in the electron temperature gradient. This may occur due to the higher density of ions on centerline retarding the flow of high-energy electrons toward the anode. It follows that this effect would be less pronounced along the walls because of increased ion losses resulting from a longer acceleration region ${ }^{18}$ and because of wall current effects. ${ }^{19}$

One prominent feature that is virtually nonexistent at 10 A is the plateau in the potential profile seen at 5.4 A. This plateau is most prominent along the inner wall where the potential exhibits a local increase in the acceleration region near the exit plane. The magnitude of this perturbation decreases from the inner wall, where the radial magnetic field is strongest, to the outer wall, where the radial magnetic field is lower. Measurements of floating potential with a cold probe showed similar results. At 5.4 A, the cold probe floating potential exhibited a local increase near the exit plane while at $10 \mathrm{~A}$ no such increase was observed. This implies that the observed plateau is an inherent feature of the plasma and not an artifact of the emitted electrons from the emissive

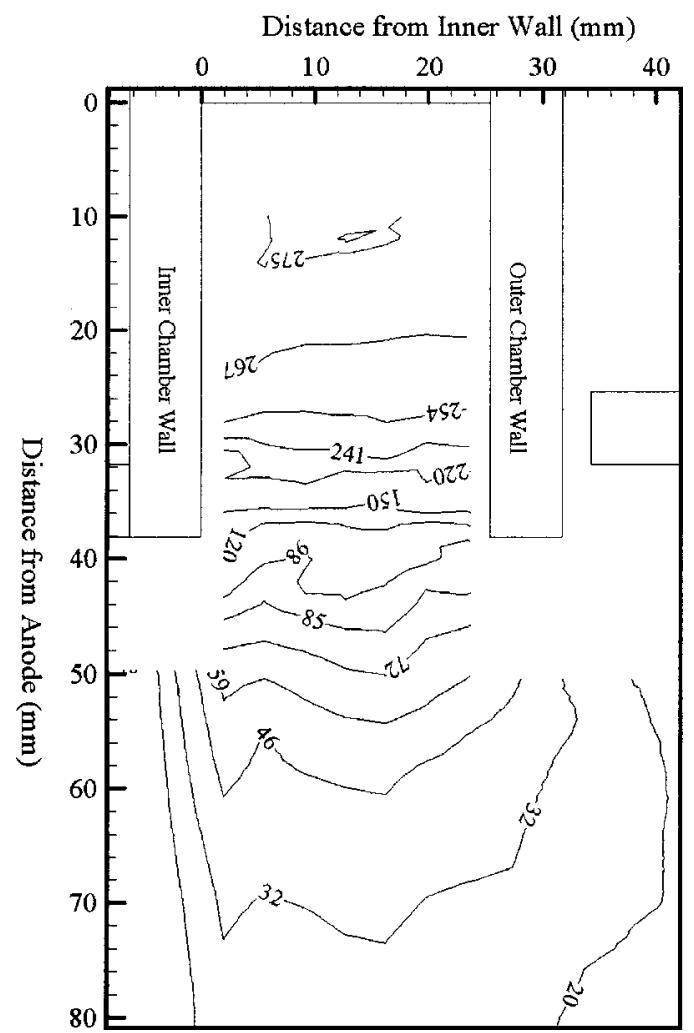

FIG. 10. Plasma potential contours at a thruster operating condition of 300 $\mathrm{V}, 5.4$ A. The maximum potential is $280 \mathrm{~V}$ with respect to ground and occurs $10 \mathrm{~mm}$ from the anode, which is at a potential of $280 \mathrm{~V}$. The potential drops sharply several millimeters upstream of the exit plane $(38 \mathrm{~mm})$ and continues to drop out to an axial position of $100 \mathrm{~mm}$. This axial profile is uniform across the width of the discharge chamber.

probe. It is believed that this perturbation may be the result of turbulence in the plasma flow as it expands out of the channel. However, a satisfactory explanation for this effect is not available at this time. It is worth noting that this feature has been observed, to a lesser extent, in other laboratory Hall thrusters as well. ${ }^{15}$

Figures 10 and 11 are the complete set of plasma potential contours. Although Figs. 8 and 9 show only three representative profiles within the radial confines of the discharge channel, a total of seven axial sweeps were performed to improve the spatial resolution of the potential map. In addition, axial sweeps were performed at radial positions beyond the inner and outer walls to investigate ion acceleration further outside the channel. All of these sweeps were combined to generate a two-dimensional contour of the plasma potential. Figure 10 shows the plasma potential contours for the $300 \mathrm{~V}, 5.4 \mathrm{~A}$ case. It is not as obvious from Fig. 10 that a local plateau occurs just downstream of the exit plane but the radial uniformity is clear. Beyond the radial confines of the discharge channel, the potential contours show significant curvature both outward and inward toward the thruster centerline. This indicates that the ions experience a radial acceleration, in addition to their considerably larger axial acceleration. Ion energy distribution measurements at the same conditions by Gulczinski ${ }^{20}$ using a molecular beam mass spectrometer (MBMS) have shown significant populations of energetic ions at large angles off centerline in both the near- 


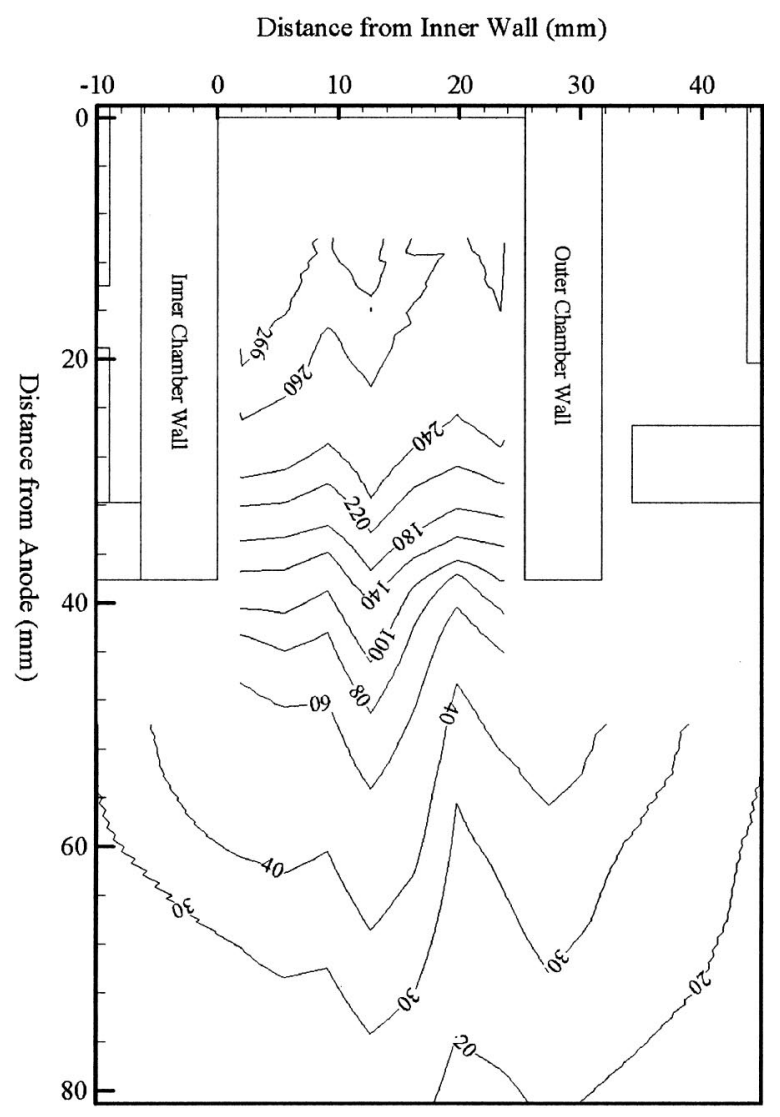

FIG. 11. Plasma potential contours at a thruster operating condition of 300 $\mathrm{V}, 10 \mathrm{~A}$. The maximum potential is $277 \mathrm{~V}$ with respect to ground and occurs $10 \mathrm{~mm}$ from the anode, which is at a potential of $278 \mathrm{~V}$. The potential drops sharply several millimeters upstream of the exit plane $(38 \mathrm{~mm})$ near the inner and outer walls but has a sharp drop downstream of the exit plane at the centerline. Note that this case lacks the radial uniformity of the $5.4 \mathrm{~A}$ case.

field (10 $\mathrm{cm}$ downstream) and far-field (75 $\mathrm{cm}$ downstream) regions. Further analysis of the near-field ion energy distributions led to the conclusion that the ions seen by the MBMS had been accelerated radially inward from the opposite side of the thruster, passing through the high-density thruster discharge. The contours of Fig. 10 show both an inward and outward curvature, however, the contours are much more sharply curved inward, suggesting ions accelerated inward pick up a larger radial component. This supports the conclusion, drawn from the MBMS data, that the nearfield ion energy distributions at large angles off centerline are dominated by ions accelerated from the opposite side of the thruster.

Figure 11 shows the plasma potential contours for the $300 \mathrm{~V}, 10$ A case. As mentioned previously, one way in which the plasma potentials of the 10 A case differ from those of the 5.4 A case is that the peak electric field on center line at $10 \mathrm{~A}$ is shifted downstream of the exit plane. This is readily apparent in Fig. 11 where a potential "jet" occurs on the centerline of the discharge channel. Additional data at 10 and $8 \mathrm{~A}$, not included in this paper, show this structure to be repeatable and evolving, becoming more pronounced as the discharge current is increased. This structure results in significant divergence of the ions as they are accelerated out of

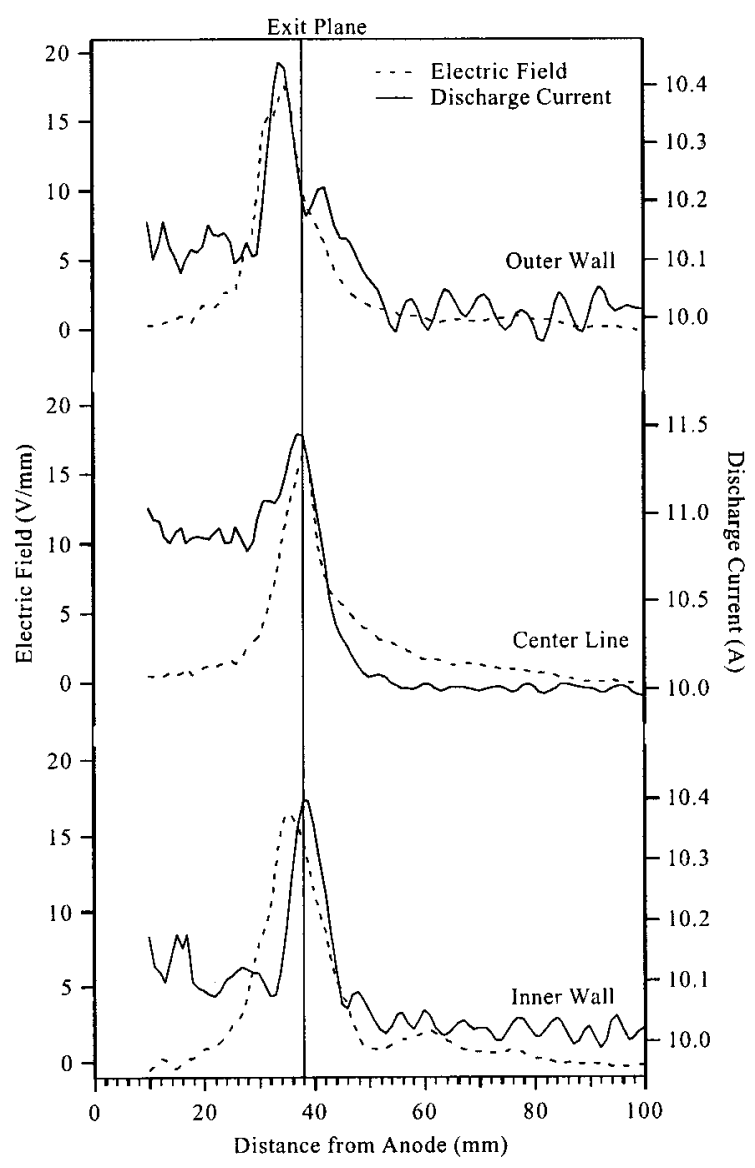

FIG. 12. Perturbations in the discharge current as a function of probe position compared to the axial electric field profiles for the $10 \mathrm{~A}$ case. Profiles for the 5.4 A case showed the same correspondence.

the thruster. LIF ${ }^{12}$ data at this condition reflect the increased divergence with a $50 \%$ increase in radial ion velocity (both inward and outward) compared to the 5.4 A case.

The discharge current was not monitored during emissive probe measurements at $5.4 \mathrm{~A}$. However, at $10 \mathrm{~A}$, the discharge current was monitored for each axial probe sweep and a 5\%-15\% variation in magnitude was observed. While zero perturbation would have been preferred, the current perturbations, when combined with the plasma potential profiles, yielded some unexpected insights. Figure 12 shows three representative traces of the discharge current and the computed axial electric field along the inner wall, outer wall, and on centerline. Only three data sets are shown here, however, each axial sweep across the discharge chamber showed the same results.

From Fig. 12, perturbations to the discharge current are seen to correspond very well to the spatial location of the peak axial electric field. Power deposition to the probe is proportional to the third power of the $\mathbf{E} \times \mathbf{B}$ drift velocity, while the $\mathbf{E} \times \mathbf{B}$ drift velocity is proportional to the magnitude of the electric field. Therefore, it is expected that the probe would experience the greatest heat load where the electric field has its largest magnitude. It follows immediately that this would be the point of greatest probe material ablation and hence discharge current perturbation. However, if this were the only mechanism disturbing thruster opera- 


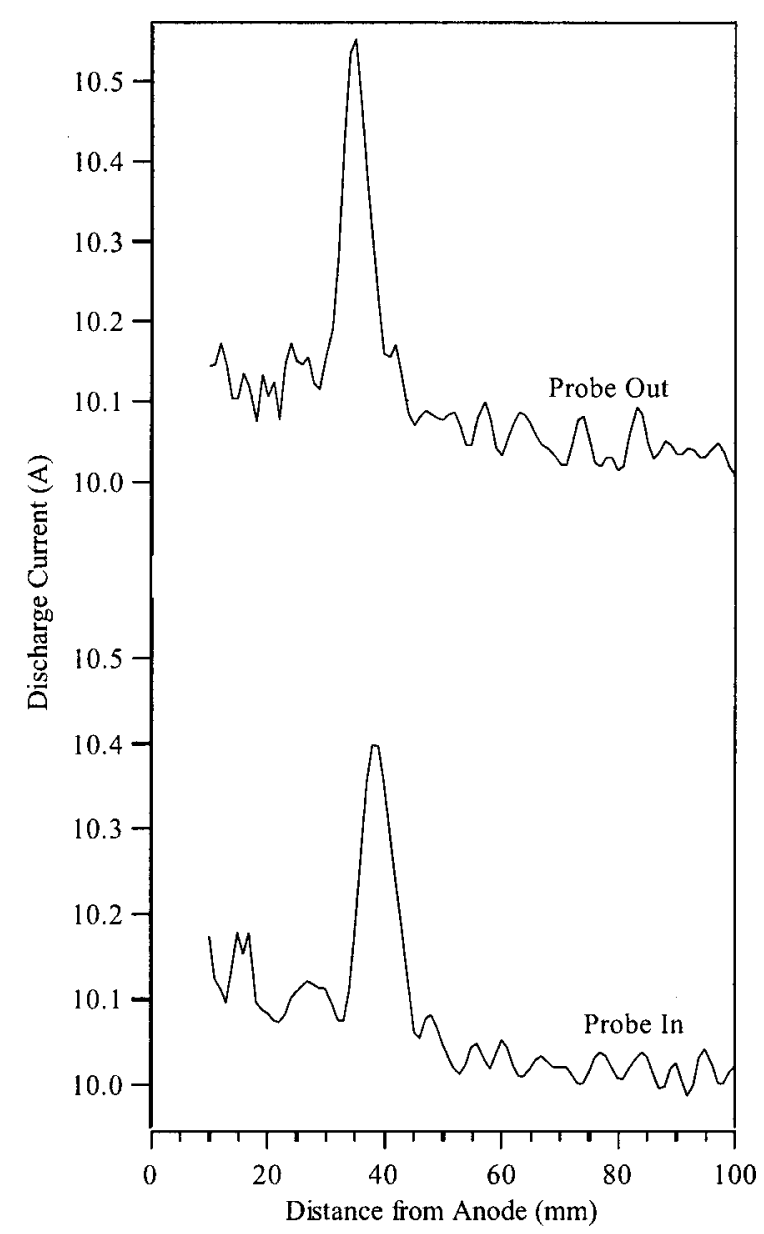

FIG. 13. Discharge current as a function of probe position inside the discharge chamber. Data are for the 10 A case.

tion, once the probe was inserted into the discharge chamber, a portion of the probe would always receive this heat load, and probe material would continue to ablate. In this case, the discharge current would remain artificially high while the probe was present inside the discharge chamber. This would also be the case if the probe were simply acting as a physical barrier, impeding the motion of the electrons as they drifted azimuthally inside the thruster.

Figure 13 shows a representative profile of the discharge current including both the insertion and removal of the probe. Recall that the probe assembly is $152 \mathrm{~mm}$ long with the emitting tungsten filament loop extending approximately $1 \mathrm{~mm}$ outside the alumina insulator. The axial position in Fig. 13 (as in all figures in this paper) corresponds to the tip of the filament. Therefore, the greatest perturbation of the discharge current occurs when the tungsten filament is coincident with the peak axial electric field. This implies one of two things; either the presence of a conductor disturbs the drift current, or the emitted electrons are somehow coupling with the drifting electrons, resulting in an increased discharge current. The data do not allow for a definitive conclusion, however, it is clear that the Hall current plays an important role in thruster operation and thruster/probe interactions and must be taken into consideration during any future internal measurements.

\section{SUMMARY}

Plasma potential inside a laboratory-model Hall thruster has been measured for two discharge current conditions. While not sufficient to fully describe the physics of the Hall thruster, the data presented have provided necessary information pertaining to the acceleration region of the thruster. At both conditions, the potential has been shown to remain nearly constant over the first $75 \%$ of the discharge channel, dropping sharply near the exit plane. At $5.4 \mathrm{~A}$, the potential remains uniform across the width of the discharge channel and the electric field indicates that ion acceleration occurs mainly inside the channel, near the exit plane, over a length of several millimeters. At $10 \mathrm{~A}$, the potential profile is no longer radially uniform and has shifted downstream. The electric field is approximately $25 \%$ lower than at $5.4 \mathrm{~A}$ indicating a longer acceleration zone and implying more ion loss to the walls. However, the data show that a larger fraction of the total acceleration occurs outside the thruster at $10 \mathrm{~A}$, where wall losses are not an issue. This provides a possible explanation for the thruster operating at higher efficiency at higher power even though the acceleration zone is longer. Perturbations to the discharge current were shown to occur when the emitting filament and peak electric field were spatially concomitant indicating a perturbation mechanism other than probe material ablation.

Mapping of the location and magnitude of the acceleration zone has provided a necessary first step toward a better understanding of Hall thruster physics. Future work will involve additional plasma parameter measurements, specifically ion number density and electron temperature, in order to provide information on the ionization zone. This information is vital in determining where ions are born inside the thruster and will indicate both how much of the accelerating potential the ion experiences and to what extent ions will be lost to the walls.

\section{ACKNOWLEDGMENTS}

The research contained herein was sponsored by the Air Force Office of Scientific Research under Dr. Mitat Birkan; this support is gratefully acknowledged. The authors would like to thank Dr. Sergei Khartov of the Moscow Aviation Institute (MAI) for providing the cathode used in these tests. They would also like to thank their fellow researchers at PEPL for assistance during experimental setup and operation. J. H. is supported by the United States Air Force Palace Knight Program.

${ }^{1}$ B. Arkhipov, A. Bober, M. Day, R. Gnizdor, K. Kozubsky, and N. Maslennikov, AIAA Paper No. 96-2708 (1996).

${ }^{2}$ A. D. Gallimore, B. E. Gilchrist, L. B. King, S. G. Ohler, and A. B. Ruffin, AIAA Paper No. 96-3298 (1996).

${ }^{3}$ D. H. Manzella, C. Sarmiento, J. M. Sankovic, and T. Haag, IEPC Paper No. 97-059, 25th International Electric Propulsion Conference, August 1997 (unpublished).

${ }^{4}$ S. O. Tverdokhlebov and V. I. Garkusha, IEPC Paper No. 97-023, 25th International Electric Propulsion Conference, August 1997 (unpublished).

${ }^{5}$ J. M. Sankovic, D. H. Manzella, and M. F. Osborn, IEPC Paper No. 97-102, 25th International Electric Propulsion Conference, August 1997 (unpublished). 
${ }^{6}$ C. E. Garner, J. R. Brophy, J. E. Polk, and L. C. Pless, AIAA Paper No. 95-2667 (1995).

${ }^{7}$ J. M. Haas, F. S. Gulczinski, A. D. Gallimore, G. G. Spanjers, and R. A. Spores, AIAA Paper No. 98-3503 (1998).

${ }^{8}$ J. M. Haas, R. R. Hofer, and A. D. Gallimore, AIAA Paper No. 99-2426 (1999).

${ }^{9}$ S. Qarnain and D. Hastings, IEPC Paper No. 97-133, 25th International Electric Propulsion Conference, August 1997 (unpublished).

${ }^{10}$ J. M. Fife, "Two-dimensional hybrid particle-in-cell modeling of Hall thrusters,"' M.S. thesis, MIT Department of Aeronautics and Astronautics, Cambridge, MA, 1995.

${ }^{11}$ N. Hershkowitz and M. H. Cho, J. Vac. Sci. Technol. A 6, 2054 (1988)

${ }^{12}$ R. F. Kemp and J. M. Sellen, Jr., Rev. Sci. Instrum. 37, 455 (1966).

${ }^{13}$ W. A. Hargus, Jr. and M. A. Cappelli, AIAA Paper No. 99-2721 (1999).
${ }^{14}$ G. J. Williams, T. B. Smith, F. S. Gulczinski, B. E. Beal, A. D. Gallimore, and R. P. Drake, AIAA Paper No. 99-2424 (1999).

${ }^{15}$ W. A. Hargus, R. J. Credolin, N. B. Meezan, and M. A. Cappelli, IEPC Paper No. 97-058, 25th International Electric Propulsion Conference, September 1997 (unpublished).

${ }^{16}$ V. I. Baranov, Yu. S. Nazarenko, V. A. Petrosov, A. I. Vasin, and Yu. M. Yashnov, IEPC Paper No. 97-060, 25th International Electric Propulsion Conference, September 1997 (unpublished).

${ }^{17}$ E. Ahedo and M. Martinez-Sanchez, AIAA Paper No. 98-8788 (1998).

${ }^{18}$ Y. Raitses, J. Ashkenazy, and G. Appelbaum, AIAA Paper No. 98-3640 (1998).

${ }^{19}$ V. Kim, J. Propul. Power 14, 736 (1998).

${ }^{20}$ F. S. Gulczinski, R. R. Hofer, and A. D. Gallimore, AIAA Paper No. 99-2430 (1999). 